\title{
Characterization and subcellular localization of histone deacetylases and their roles in response to abiotic stresses in soybean
}

Chao Yang ${ }^{1,2+}$, Wenjin Shen ${ }^{2 \dagger}$, Hongfeng Chen ${ }^{1}$, Liutian $\mathrm{Chu}^{1,3}$, Yingchao Xu ${ }^{1,3}$, Xiaochen Zhou ${ }^{1,3}$, Chuanliang Liư ${ }^{2}$, Chunmiao Chen ${ }^{2}$, Jiahui Zeng ${ }^{2}$, Jin Liu', Qianfeng Li ${ }^{5}$, Caiji Gao ${ }^{2}$, Jean-Benoit Charron ${ }^{6}$ and Ming Luo ${ }^{1 *}$ (D)

\begin{abstract}
Background: Histone deacetylases (HDACs) function as key epigenetic factors in repressing the expression of genes in multiple aspects of plant growth, development and plant response to abiotic or biotic stresses. To date, the molecular function of HDACs is well described in Arabidopsis thaliana, but no systematic analysis of this gene family in soybean (Glycine max) has been reported.

Results: In this study, 28 HDAC genes from soybean genome were identified, which were asymmetrically distributed on 12 chromosomes. Phylogenetic analysis demonstrated that GmHDACs fall into three major groups previously named RPD3/HDA1, SIR2, and HD2. Subcellular localization analysis revealed that YFP-tagged GmSRT4, GmHDT2 and GmHDT4 were predominantly localized in the nucleus, whereas GmHDA6, GmHDA13, GmHDA14 and GmHDA16 were found in both the cytoplasm and nucleus. Real-time quantitative PCR showed that GmHDA6, GmHDA13, GmHDA14, GmHDA16 and GmHDT4 were broadly expressed across plant tissues, while GmHDA8, GmSRT2, GmSRT4 and GMHDT2 showed differential expression across various tissues. Interestingly, we measured differential changes in GmHDACs transcripts accumulation in response to several abiotic cues, indicating that these epigenetic modifiers could potentially be part of a dynamic transcriptional response to stress in soybean. Finally, we show that the levels of histone marks previously reported to be associated with plant HDACs are modulated by cold and heat in this legume.
\end{abstract}

Conclusion: We have identified and classified 28 HDAC genes in soybean. Our data provides insights into the evolution of the HDAC gene family and further support the hypothesis that these genes are important for the plant responses to environmental stress.

Keywords: Histone deacetylases, Subcellular localization, Gene expression, Abiotic stresses, Soybean

\section{Background}

Throughout their life course, plants are frequently exposed to suboptimal environmental conditions that cause adverse effects on their growth and development. Abiotic stress is one of the major causes of agricultural losses in the world [1,2]. Soybean (Glycine max) is an important global crop desirable for its high protein

\footnotetext{
* Correspondence: luoming@scbg.ac.cn

${ }^{\dagger}$ Chao Yang and Wenjin Shen contributed equally to this work.

${ }^{1}$ Guangdong Provincial Key Laboratory of Applied Botany, Key Laboratory of South China Agricultural Plant Molecular Analysis and Genetic Improvement, South China Botanical Garden, Chinese Academy of Sciences, Guangzhou 510650, China

Full list of author information is available at the end of the article
}

content and oil. More than $50 \%$ of globally consumed edible oil is contributed by soybeans while the proteins of this legume are highly desirable for food and feed applications [3]. However, the production of soybeans is greatly affected by abiotic stresses such as salt, drought, cold, heat, water submergence and heavy metals [3]. Recently, studies have demonstrated that epigenetic processes play vital regulatory roles in plant abiotic stress responses [4-7]. Chromatin structure and gene expression changes in response to abiotic stress are controlled through epigenetic mechanisms such as histone modification and chromatin remodeling. Among them, histone

(c) The Author(s). 2018 Open Access This article is distributed under the terms of the Creative Commons Attribution 4.0 International License (http://creativecommons.org/licenses/by/4.0/), which permits unrestricted use, distribution, and reproduction in any medium, provided you give appropriate credit to the original author(s) and the source, provide a link to the Creative Commons license, and indicate if changes were made. The Creative Commons Public Domain Dedication waiver (http://creativecommons.org/publicdomain/zero/1.0/) applies to the data made available in this article, unless otherwise stated. 
acetylation is regulated by histone acetyltransferases (HATs) and histone deacetylases (HDACs).

In the past decades, a large number of HDACs have been identified and characterized in plants, which can be grouped into three different families: the RPD3/HDA1, the SIR2, and the HD2. Members of the RPD3/HDA1 and the SIR2 families are proteins homologous to the yeast Reduced Potassium Dependency 3 (RPD3)/HDA1 and Silent Information Regulator 2 (SIR2), respectively, whereas the HD2 family was originally characterized in maize and appears to be present only in plants [8-10]. Members of the SIR2 family have a catalytic domain that requires nicotine adenine dinucleotide (NAD) as a cofactor [11], while members of the RPD3/HDA1 family share HDAC domain sequence homology and require a $\mathrm{Zn}^{2+}$ cofactor for deacetylase activity [9]. The HD2 family proteins contain a conserved pentapeptide motif (MEFWG) at the $\mathrm{N}$-terminus and are considered to be zinc-dependent HDACs [12].

The Arabidopsis HDACs have been well characterized. The genome of this model plant encodes 18 HDACs distributed into the three aforementioned HDAC families $[10,13]$. Increasing evidence suggests that AtHDACs play essential roles in regulating multiple aspects of plant growth and development $[4,14,15]$. AtHDA6 is a member of the RPD3/HDA1 family that was originally reported to play an important role in coordinating downstream gene silencing and maintenance of DNA methylation [16, 17]. It was also demonstrated that HDA6 interacted with a number of protein partners, such as FLOWERING LOCUS D (FLD), ASYMMETRIC LEAVES 1 (AS1), TOPLESS (TPL), and JASMONATE ZIM-DOMAIN (JAZ) to regulate flowering, leaf development, circadian transcription and hormonal responses by modulating the transcription of their respective target genes through histone deacetylation [18-21]. Other members of the RDP3/HDA1 family, such as AtHDA5, AtHDA7, AtHDA9, AtHDA14, AtHDA15, AtHDA18 and AtHDA19, were also reported to play important roles in plant growth and development [22-37]. For example, hda19 mutants displayed various developmental abnormalities, such as early senescence, suppression of apical dominance, flower defects, and male and female sterility, indicative of the importance of AtHDA19 for proper vegetative development [22, 24, 31]. HDA5 forms a protein complex with HDA6, FLD and MSI1-like WD40 repeat 4 (FVE/MSI4) in regulation of flowering time by repressing FLOWERING LOCUS C (FLC) expression through histone deacetylation [34]. The BRI1-EMSSUPPRESSOR1 (BES1)-TPL-HDA19 repressor complex is required for deacetylation of ABA-insensitive 3 (ABI3) gene in controlling early seedling development [32]. Additionally, members of the HD2 family play crucial roles in plant development [38-42]. For instance, silencing of
HD2A in Arabidopsis resulted in aborted seed development [38], while overexpression of $H D 2 A$ caused morphological defects of leaves and flowers, delayed flowering and aborted seed development [39]. It was demonstrated that the SIR2 family protein AtSRT2 plays a negative role in plant basal defense against the pathogen PstDC3000, and the expression of AtSRT2 was repressed in response to the pathogen infection [43]. In rice (Oryza sativa), OsSRT1 is required for repressing the expression of starch metabolism genes during seed development [44].

In Arabidopsis, AtHDACs play vital roles in plant responses to abiotic and biotic stresses [4, 45-53]. It was reported that AtHDACs were responsive to various environmental cues at the transcription level [13], for example, AtHDA6 and AtHDA19 are induced by JA treatment [45], whereas the expression of $H D 2 A, H D 2 B$, $H D 2 C$, and $H D 2 D$ is repressed by $\mathrm{ABA}$ and $\mathrm{NaCl}[49$, 54]. AtHDA9 was shown to negatively regulate high salt and drought responses [53]. On the other hand, AtHDA6, AtHD2C, and AtHD2D confer plant resistance under high salt, drought, cold or freezing conditions [49-51, 55]. Recently, emerging evidence indicates that hda19 plants exhibit tolerance to high salinity stress, while hda5/14/15/18 plants exhibit hypersensitivity to salt stress. This suggests that AtHDA19 and AtHDA5/ $14 / 15 / 18$ control plant responses to salt stress in different pathways [53]. While, different members of the HDAC family have been found to mediate distinct aspects of the plant growth and development as well as its abiotic and biotic stress responses, the characterization of $H D A C$ genes in soybean has not been reported yet.

In this study, we have identified $28 H D A C$ coding genes in the soybean genome. YFP-tagged transient expression assays confirmed the subcellular localization of GmHDACs. While tissue-specific and stress-responsive expression patterns for nine representative genes were determined using quantitative RT-PCR. In addition, the levels of histone acetylation and methylation were analyzed under cold and heat treatments. Together, our results shed light on the involvement of GmHDAC genes in various aspects of plant growth and development including the response to abiotic stress.

\section{Results \\ Identification and phylogenetic analysis of soybean histone deacetylases}

The protein sequences of AtHDACs were used as queries to conduct sequence homology searches against the Soybase database (https://soybase.org/). In total, 28 independent soybean GmHDACs were identified. (Table 1). The complete open reading frames (ORFs) of the retrieved GmHDAC genes ranged from 582 to $1971 \mathrm{bp}$, while predicted proteins ranged from 193 to 656 amino acids with calculated molecular weights from 21.37 to 
Table 1 Overview of histone deacetylases genes identified in soybean

\begin{tabular}{|c|c|c|c|c|c|c|c|c|c|}
\hline \multicolumn{3}{|l|}{ Gene ID } & \multicolumn{3}{|l|}{ DNA attributes } & \multicolumn{4}{|c|}{ Protein attributes } \\
\hline$\overline{\text { Gene name }^{a}}$ & Gene locus $^{\mathrm{b}}$ & Accession number ${ }^{c}$ & Chromosome & ORF (bp) & No. of exons & Length (aa) & $\mathrm{MW}(\mathrm{kDa})$ & $\mathrm{Pl}$ & Localization \\
\hline GmHDA1 & Glyma.01 g245100 & XP_003517607.1 & 1 & 1494 & 7 & 497 & 56.28 & 5.30 & nucl, cyto \\
\hline GmHDA2 & Glyma.04 g000200 & XP_003543935.1 & 4 & 1494 & 7 & 497 & 55.96 & 5.04 & cyto, nucl \\
\hline GmHDA3 & Glyma.04 g187000 & XP_006578653.1 & 4 & 1398 & 6 & 465 & 52.18 & 5.28 & cyto, chlo \\
\hline GmHDA4 & Glyma.04 g187100 & KRH63610.1 & 4 & 594 & 2 & 197 & 22.26 & 6.31 & cyto \\
\hline GmHDA5 & Glyma.05 g012900 & KRH56687.1 & 5 & 1254 & 14 & 417 & 45.28 & 7.39 & nucl, cyto \\
\hline GmHDA6 & Glyma.05 g021400 & XP_003524633.1 & 5 & 1971 & 14 & 656 & 73.04 & 5.25 & cyto, nucl \\
\hline GmHDA7 & Glyma.05 g040600 & XP_003525556.1 & 5 & 1431 & 6 & 476 & 53.30 & 5.24 & nucl \\
\hline GmHDA8 & Glyma.05 g192600 & XP_014631275.1 & 5 & 1263 & 9 & 420 & 45.54 & 6.09 & chlo, nucl \\
\hline GmHDA9 & Glyma.06 g000100 & XP_003526730.1 & 6 & 1494 & 7 & 497 & 55.95 & 5.06 & nucl, cyto \\
\hline GmHDA10 & Glyma.06 g178700 & KRH54335.1 & 6 & 705 & 1 & 234 & 26.74 & 8.88 & chlo, cyto \\
\hline GmHDA11 & Glyma.11 g000300 & XP_006590384.1 & 11 & 1494 & 7 & 497 & 56.12 & 5.22 & nucl, cyto \\
\hline GmHDA12 & Glyma.11 g187800 & XP_003538135.1 & 11 & 1290 & 14 & 429 & 48.94 & 4.98 & cyto, nucl \\
\hline GmHDA13 & Glyma.12 g086700 & XP_003539814.1 & 12 & 1290 & 14 & 429 & 48.94 & 5.06 & cyto, mito \\
\hline GmHDA14 & Glyma.12 g188200 & XP_003540263.1 & 12 & 1146 & 3 & 381 & 41.18 & 5.44 & cyto, chlo \\
\hline GmHDA15 & Glyma.17 g078000 & KRH03123.1 & 17 & 1971 & 14 & 656 & 72.99 & 5.35 & cyto, nucl \\
\hline GmHDA16 & Glyma.17 g085700 & XP_003549603.1 & 17 & 1419 & 6 & 472 & 52.92 & 5.26 & nucl, chlo \\
\hline GmHDA17 & Glyma.17 g120900 & XP_006600776.1 & 17 & 1632 & 17 & 543 & 59.6 & 5.91 & nucl, cyto \\
\hline GmHDA18 & Glyma.17 g229600 & XP_003550277.1 & 17 & 1047 & 13 & 348 & 38.50 & 6.29 & cyto, nucl \\
\hline GmSRT1 & Glyma.04 g210000 & XP_003522478.1 & 4 & 1182 & 11 & 393 & 43.46 & 9.40 & chlo, mito \\
\hline GmSRT2 & Glyma.06 g156000 & XP_003528059.2 & 6 & 1179 & 11 & 392 & 43.21 & 9.32 & chlo, mito \\
\hline GmSRT3 & Glyma.08 g330200 & KHN11152.1 & 8 & 1302 & 13 & 433 & 48.09 & 9.11 & chlo, nucl \\
\hline GmSRT4 & Glyma.18g076300 & XP_003551434.1 & 18 & 1440 & 14 & 479 & 53.19 & 9.15 & nucl, cyto \\
\hline GmHDT1 & Glyma.03 g190700 & NP_001240859.1 & 3 & 867 & 10 & 288 & 31.46 & 4.64 & nucl \\
\hline GmHDT2 & Glyma.11 g189500 & XP_006591094.1 & 11 & 870 & 9 & 289 & 30.77 & 4.80 & nucl \\
\hline GmHDT3 & Glyma.12 g084700 & $\mathrm{KRH} 25160.1$ & 12 & 900 & 9 & 299 & 31.77 & 4.75 & nucl \\
\hline GmHDT4 & Glyma.12 g181400 & $\mathrm{KRH} 26575.1$ & 12 & 924 & 10 & 307 & 33.31 & 4.9 & nucl \\
\hline GmHDT5 & Glyma.13 g319500 & $\mathrm{KRH} 22734.1$ & 13 & 582 & 6 & 193 & 21.37 & 4.29 & chlo, cyto \\
\hline GmHDT6 & Glyma.19 g191000 & XP_003554417.1 & 19 & 882 & 9 & 293 & 31.91 & 4.61 & nucl \\
\hline
\end{tabular}

${ }^{a}$ Systematic designation given to soybean histone deacetylase genes

${ }^{b}$ Accession number of Soybase (http://soybase.org/) locus ID

'Accession numbers of protein sequence available at NCBI (http:// www.ncbi.nlm.nih.gov/)

${ }^{d}$ Subcellular Localization of soybean histone deacetylases supported by WoLF PSORT (http://www.genscript.com/psort/wolf_psort.html)

CDS, coding sequence; No., number; MW, molecular weight; $\mathrm{Pl}$, isoelectric point

Nuc, nuclear; Cyto, cytoplasm; Chl, chloroplast; Mito, mitochondrion

$72.99 \mathrm{kDa}$ and isoelectric points from 4.29 to 9.40 (Table 1). Further bioinformatics analyses indicated that GmHDACs were potentially localized in several organelles such as the nucleus, cytoplasm, chloroplast and mitochondria (Table 1).

To evaluate the evolutionary relationship of plant histone deacetylases, a phylogenetic analysis was performed using the protein sequences of HDACs from soybean and Arabidopsis. The phylogenetic tree indicated that the 28 newly uncovered soybean HDACs are grouped into three types characterized by distinctive protein structures (Table 1 and Fig. 1). In soybean, type I (RPD3/HDA1 family) HDACs consist of 18 members, named GmHDA1 to GmHDA18 based on their coordinates on soybean chromosome (Table 1). All 18 members of this type have a characteristic histone deacetylase domain (Interpro: IPR003084) (Fig. 1b) and can be further divided into four classes based on sequence similarity (Fig. 1a). Class I encompassed 11 GmHDACs, Class II included 5 members while classes III and IV each contained one GmHDAC (Fig. 1a). The phylogenetic analysis also demonstrated that soybean has four type II (SIR2 family) HDACs with highly conserved Sir2 domains (Table 1 and Fig. 1). Finally, six plant-specific HDACs (type III: HD2 family) with conserved N-terminal MEFWG amino acid regions (Fig. 1b), which is required 


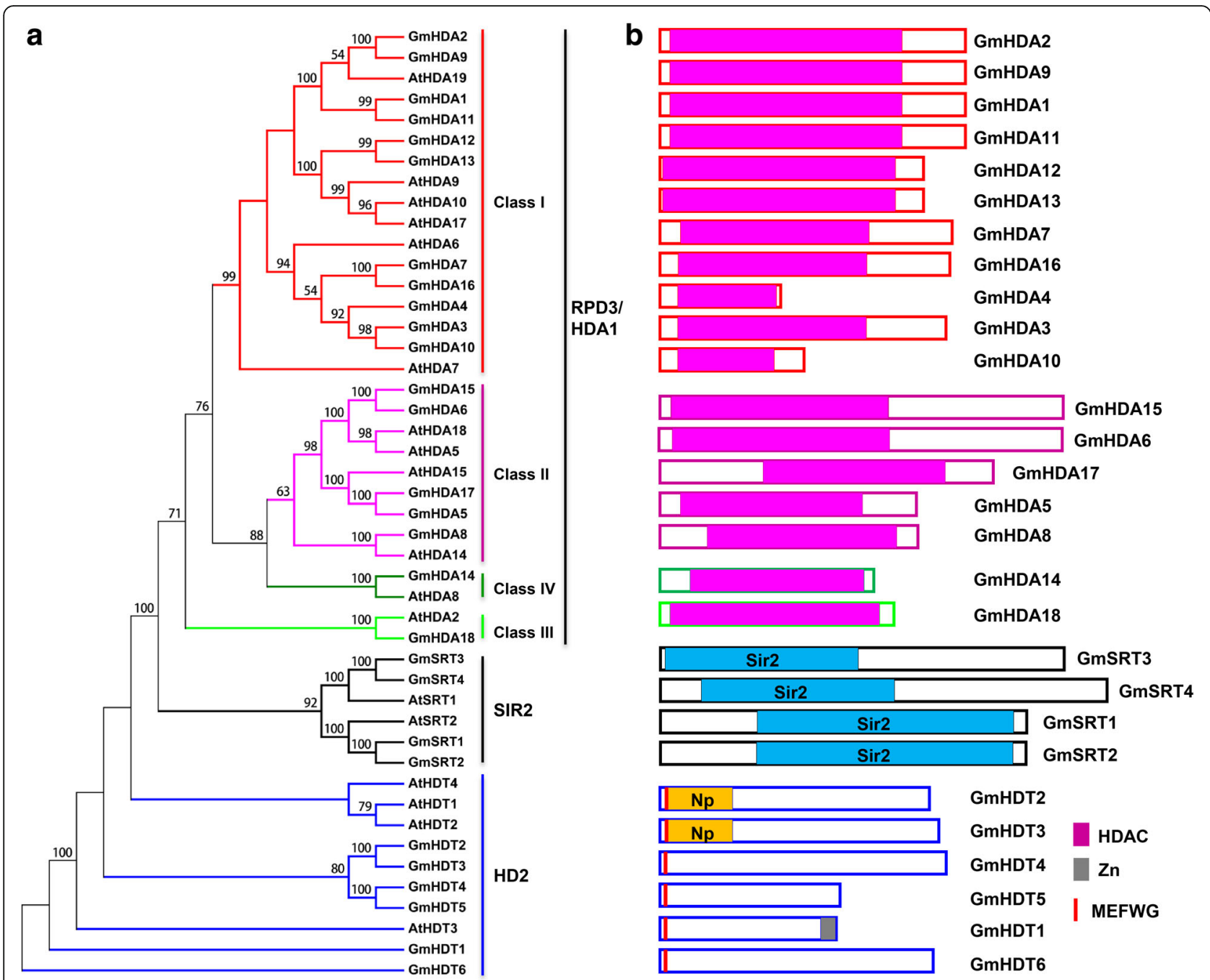

Fig. 1 Phylogenetic tree and domain architecture of HDACs in soybean. a Phylogenetic tree of HDAC proteins in soybean and Arabidopsis. The phylogenetic tree was generated using the MEGA 5.0 software and the Maximum Parsimony method with the following parameters: bootstrap analysis of 1000 replicates and partial deletion. The numbers at the nodes indicate the bootstrap values. $\mathbf{b}$ The schematic diagrams show the domain organization of these proteins according to analysis by NCBI Batch-CD, SMART and PFAM searches. Different domains are indicated by the use of different colors. The proteins belonging to each family are grouped together

for transcriptional repression followed by a central acidic region rich in glutamic and/or aspartic acid [9] were identified in soybean (Table 1 and Fig. 1). A C2H2 zinc finger domain in the C-terminus portion of GmHDT1 was also detected, which might indicate that this protein has high DNA-binding affinity or could mediate protein-protein interactions. Additionally, both GmHDT2 and GmHDT3 may bind to nucleoplasmins through their $\mathrm{N}$-terminal nucleophosmin domains (Fig. 1b).

\section{Chromosomal localization and duplications of HDAC genes in soybean}

The chromosomal localization of the 28 GmHDAC genes was determined by their genomic distributions on soybean chromosomes. The 28 GmHDAC genes were asymmetrically distributed on 12 chromosomes (Fig. 2). Chromosome 4, 5, 12 and 17 each contain the largest number of $H D A C$ genes with four each, followed by chromosome 6 and 11 with three genes each, whereas only one $H D A C$ gene was present on each of chromosomes $1,3,8,13,18$, and 19. No HDAC genes were found on chromosome 2, 7, 9, 10, 14, 15, and 20 (Fig. 2). Moreover, the $H D A C$ gene density per chromosome was uneven with the highest densities of $H D A C s$ at proximal regions of chromosomes 5 and 17 and the distal region of chromosome 4 (Fig. 2).

Tandem duplication and segmental genome duplication are major contributors to the generation and maintenance of gene families. If two paralogous genes are physically close together, we conclude that they probably 


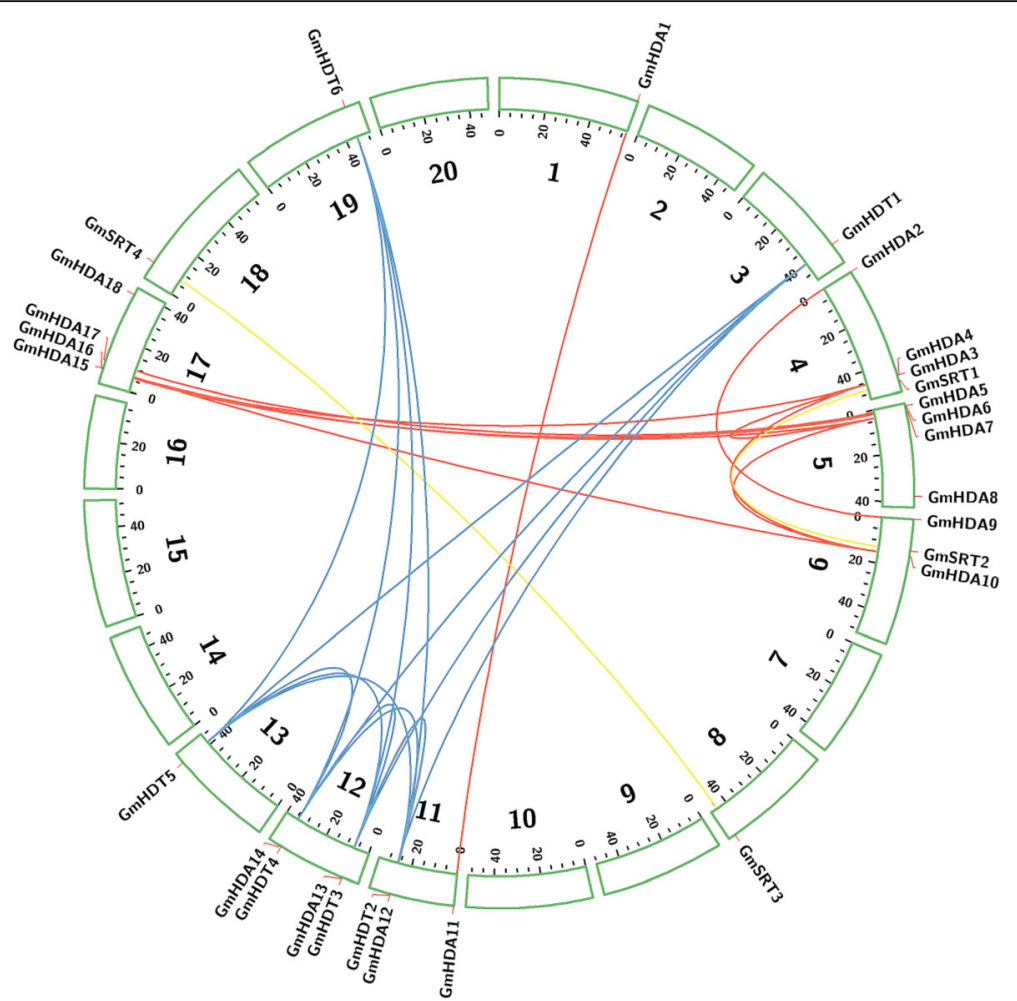

Fig. 2 Chromosomal localization and duplication of HDAC genes in soybean. Each box refers to a chromosome, and chromosome numbers are shown beside each chromosome. The scale is in megabase. The approximate location of each soybean HDAC gene is indicated by a short orange line. Colored lines in the circle indicate the linkage group with segmentally duplicated HDAC gene pairs, and segmental duplication regions were determined using the Plant Genome Duplication Database

arose through tandem duplication [56]. It was found that only one gene set (GmHDA3 and GmHDA4) was tandemly distributed on chromosome 4 and was separated by 4407 bp. However, only $23.8 \%$ and $29.7 \%$ of sequence identity at the nucleotide and protein level were respectively observed between them, indicating that GmHDA3 and GmHDA4 have not undergone tandem amplification during evolution. We further investigated whether segmental duplications contributed to the expansion of $H D A C$ gene family in soybean. It is noteworthy that 27 duplication sets of $H D A C$ genes on the same block were identified. These duplicated genes pairs clustered into a discrete clade in the phylogenetic tree and shared a high degree of identity at the protein level within each pair (Figs. 1 and 2, Additional file 1: Table S1). To evaluate the selection mode of the 27 duplicated gene pairs of HDAC in soybean, we calculated the nonsynonymous/ synonymous substitution ratios $(\mathrm{Ka} / \mathrm{Ks})$. According to the literature a $\mathrm{Ka} / \mathrm{Ks}$ ratio above 1 indicates positive selection while ratios below and equal to 1 respectively indicate purifying and neural selection [57]. As shown in the Additional file 1: Table $\mathrm{S} 1$, the $\mathrm{Ka} / \mathrm{Ks}$ values of all duplicated gene pairs were less than 1 , suggesting that these duplicates likely have been subjected to purifying selection.

\section{Subcellular localization of GmHDACs}

Prediction analysis indicated that GmHDACs exhibit various patterns of subcellular localization (Table 1). To further determine the subcellular localization of GmHDACs, full-length cDNAs were fused to the Yellow Fluorescent Protein (YFP) driven by the CaMV $35 \mathrm{~S}$ promoter and transiently expressed in protoplasts of Arabidopsis suspension culture cells. As shown in Fig. 3, four RPD3/HDA1 family members, GmHDA6, 13, 14 and 16 localized in both the cytosol and nucleus. Consistent with the predicted localization using bioinformatics programs, GmSRT4 and two members of HD2 family (GmHDT2 and GmHDT4) localized in the nucleus, mainly in the nucleolus (Fig. 3).

\section{Tissue and organ specific expression of HDAC genes in soybean}

To investigate the tissue and organ specific expression profiles of $H D A C$ genes in soybean, quantitative RT-PCR assays for nine representative $H D A C$ genes from different families were conducted in different tissues and developmental stages. As shown in Fig. 4, GmHDA6, GmHDA13, GmHDA14 and GmHDA16 were ubiquitously expressed at high levels in most of the organs examined. GmHDA8 showed high expression in cotyledon and leaf, as well as 


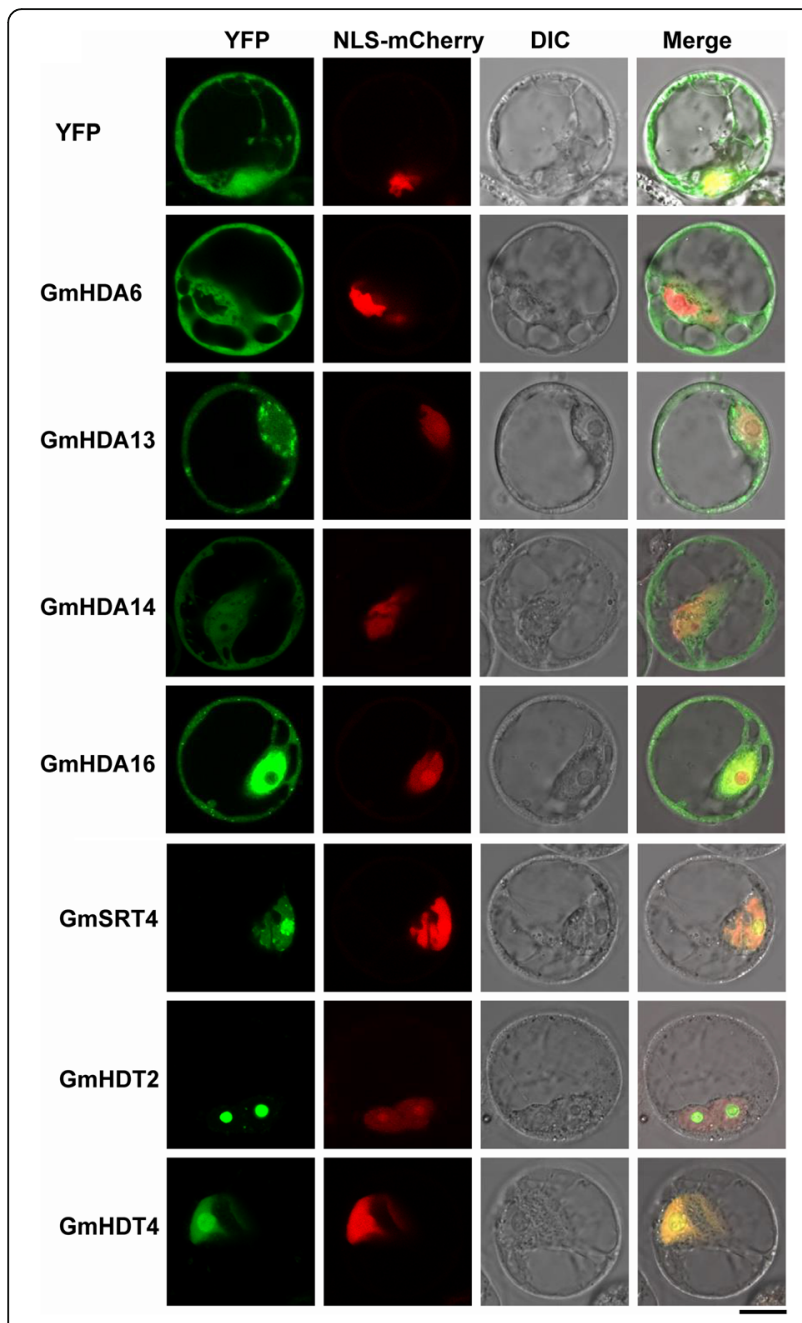

Fig. 3 Subcellular localization of GmHDACs. GmHDACs-EYFP fusion constructs were used to determine the subcellular localization of GmHDACs. NLS-mCherry was used as a nuclear marker. Fluorescence images of YFP and mCherry were captured with confocal laser scanning microscopy and are shown in green and red, respectively (scale bars, $10 \mu \mathrm{m}$ )

relatively low expression in other tissues. Specific high transcript accumulation of GmSRT2 was observed in the leaves, while the expression in the other organs remained low. Unlike GmSRT2, GmSRT4 mRNA levels were high in roots and flower tissues, moderate in hypocotyls and seeds, and lowest in the leaves. In addition, the GmHDT2 transcripts were abundant in roots and stems, whilst relatively low in other tissues. Finally, GmHDT4 was ubiquitously expressed at high levels in most of the organs examined, except for the cotyledons.

Soybean seeds are one of the most important agricultural commodities, being a prime source of oil, protein, and carbohydrate. To investigate the expression profiles of GmHDAC genes during soybean seed development and maturation, we obtained publicly available transcriptome data from the Soybase database (https://soybase.org/). As shown in Additional file 2: Fig. S1, nine GmHDAC genes displayed differential expression patterns in developing seeds. GmHDA8, GmHDA14, GmHDT2 and GmHDT4 were highly expressed throughout seed development. However, the expression of GmSRT4 was only detected at the early stages of seed development, whereas GmHDA13 was highly expressed during the middle stage. GmHDA6 transcripts accumulated progressively early in developing seeds but their abundance decreased markedly during the seed maturation process. Additionally, the transcripts of GmHDA16 were most abundant in 21 DAF (days after flowering) seeds but scarce in 28 DAF seeds (Additional file 2: Fig. S1).

GmHDACs are involved in various abiotic stress responses Evidence suggests that $H D A C s$ play important functions in plant response to various abiotic stresses. To study the potential roles of GmHDACs in abiotic stress responses, we performed expression analysis of GmHDACs under various abiotic stress conditions using quantitative RT-PCR. As shown in Fig. 5, our results demonstrated that GmHDAC genes significantly responded to various abiotic stress treatments. When exposed to cold, the expression of seven GmHDAC genes (GmHDA6, 13, 14, 16, GmSRT4, GmHDT2 and GmHDT4) was strongly repressed, while GmSRT2 expression was significantly induced. On the other hand, GmHDA8 was not significantly changed in response to low temperature (Fig. 5a). Following a heat shock treatment, five GmHDAC genes (GmHDA6, 8, 14, GmSRT4 and GmHDT4) became down-regulated, GmSRT2 was up-regulated, while the expression of GmHDA13, 16 and GmHDT2 were unchanged (Fig. 5b). Notably, the accumulation level of the GmHDA8 transcript was nearly completely suppressed under heat treatment (Fig. 5b). Furthermore, the differential expression patterns of the $G m H D A C$ genes under flooding stress were observed. Four RPD3/HDA1 family genes, GmHDA6, 8, 14 and 16 were down-regulated, while GmHDA13, GmSRT4 and GmHDT2 were slightly up-regulated and GmSRT2 and GmHDT4 did not respond to the flooding treatment (Fig. 5c). The expression levels of all nine GmHDACs examined decreased following a drought stress treatment with $G m H D A 8$, GmHDA14, GmSRT4, GmHDT2 and GmHDT4 being the most impacted (Fig. 5d). Finally, high salt treatment significantly induced GmSRT2 expression, but repressed the expression of GmHDA6, 8, 14, 16, GmSIR4, GmHDT2 and GmHDT4, and did not modulate the expression of GmHDA13 (Fig. 5e).

Finally, we evaluated the responses of GmHDACs to abscisic acid (ABA) treatment, which is the most important stress-protective phytohormone. Two HD2 family genes (GmHDT2 and GmHDT4) were induced and two RPD3/ HDA1 family genes (GmHDA8 and GmHDA16) were 


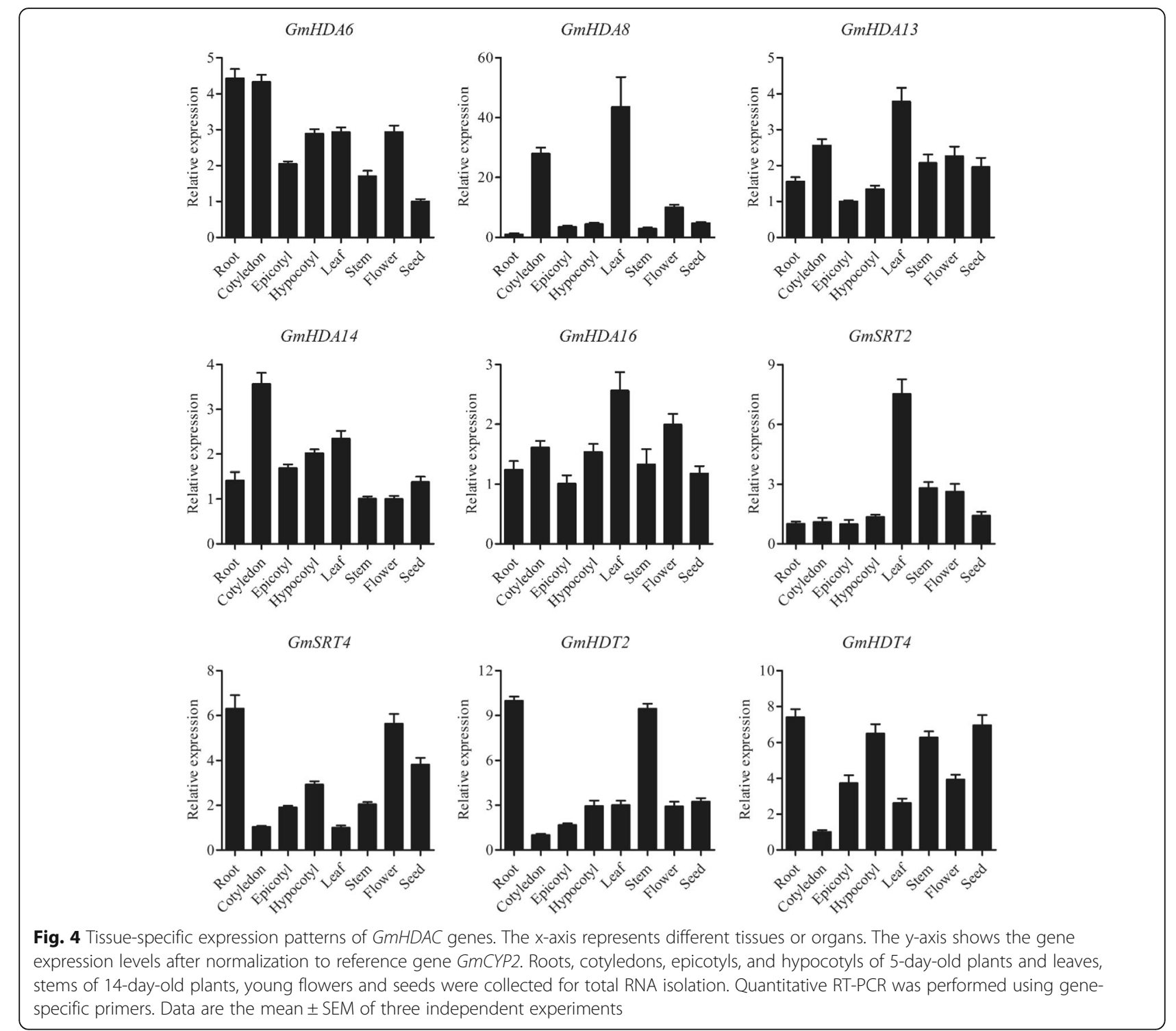

markedly suppressed by our ABA treatment. On the other hand, the expression of five other HDAC genes (GmHDA6, 13, 14, GmSRT2 and GmSRT4) was only slightly modulated (Fig. 5f). In addition, status of $\mathrm{H} 3$ acetylation following our cold and heat treatments was analyzed. As shown in Fig. 6, the level of H3ac decreased under cold treatment, but increased after heat treatment. Interestingly, status of H3K4me2 and H3K4me3 were also modulated by the both cold and heat treatments. High levels of H3K4me 2 and low levels of H3K4me3 were observed following our cold treatment. Meanwhile, the level of H3K4me2 was up-regulated in response to heat stress, while this treatment only had a mild effect on H3K4me3 levels (Fig. 6).

\section{Discussion}

Studies have shown that HDACs play critical roles in multiple aspects of plant development and response to various environmental cues by regulating gene expression through histone deacetylation $[4,14,15]$. Genome-wide identification and characterization of HDACs have previously been reported in several plant species, including Arabidopsis, rice, grape (Vitis vinifera) and tomato (Solanum lycopersicum) etc. [10, 58-61]. However, the involvement of HDACs in the response to environmental cues has not been documented in soybean. In this study, 28 HDACs were identified and characterized in soybean, with respect to their tissue-specific expression profiles, subcellular localizations and abiotic stress responsive expression patterns. The 28 GmHDACs are divided into three families: RPD3/HDA1, SIR2 and HD2 and are unevenly distributed on 12 soybean chromosomes (Figs. 1 and 2). The number of $H D A C$ gene in soybean is larger than those in Arabidopsis, rice and tomato and compared to other plant species, gene expansion of $H D A C s$ in soybean is evident 


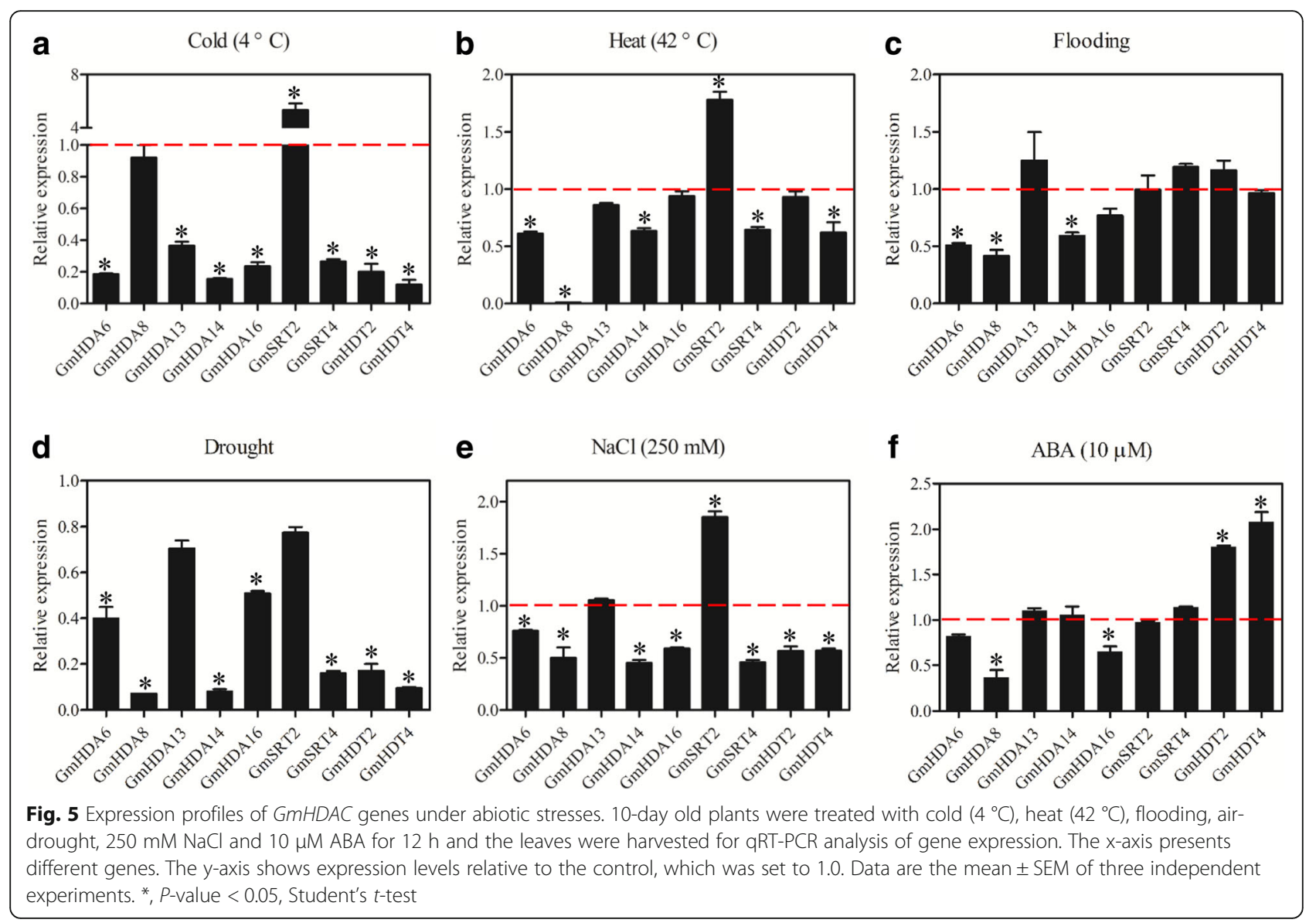

[10, 58, 60, 61]. For example, Arabidopsis, rice and tomato only have two SIR2 genes, while soybean contains four (Fig. 1a). Tandem amplification and segmental duplication of chromosomal regions are likely the main contributors for gene extension during evolution [56]. In total, $27 \mathrm{du}$ plication pairs of $H D A C$ genes were identified but no tandem gene sets were found (Fig. 2, Additional file 1: Table S1), indicating that segmental duplication events during evolution are most likely the major drivers of HDAC gene expansion in soybean.

In humans, RPD3 type Class I HDACs are localized exclusively in the nucleus, whereas class II HDACs are
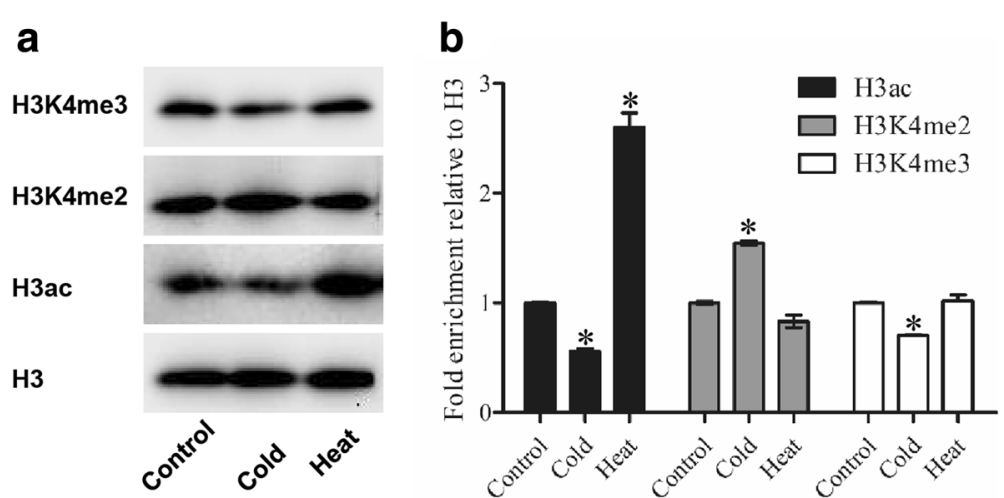

Fig. 6 Levels of histone $\mathrm{H} 3 \mathrm{ac}, \mathrm{H} 3 \mathrm{~K} 4 \mathrm{me}$, and $\mathrm{H} 3 \mathrm{~K} 4 \mathrm{me} 3$ under cold and heat treatments. a Western blot showing the H3ac, $\mathrm{H} 3 \mathrm{~K} 4 \mathrm{me2}$, and H3K4me3 status in soybean leaves treated with cold and heat. 10-day-old seedlings were treated under cold $\left(4^{\circ} \mathrm{C}\right)$ and heat $\left(42^{\circ} \mathrm{C}\right)$ conditions for $12 \mathrm{~h}$ and the leaves were sampled for total protein extraction. $\mathbf{b}$ Quantification of western blot results. Signal intensities were measured using the ImageJ software and normalized to the loaded amount of H3. Values are expressed as fold change over control treatment. Shown is the mean \pm SEM of three independent experiments. *, P-value $<0.05$, Student's $t$-test 
shuttled between the cytoplasm and the nucleus [62]. In Arabidopsis, previous studies demonstrated that RPD3 type Class II HDACs, HDA5, HDA8, and HDA14 are localized in the cytoplasm, whereas HDA15 is localized exclusively in the nucleus. In addition, AtHDA15 was shown to shuttle from the cytoplasm to the nucleus is response to light [63]. Moreover, RPD3 type Class I HDA6 and HDA19 also localized in the nucleus [45, 64, 65]. In this study, we found that GmHDA6, GmHDA13, GmHDA14 and GmHDA16 were localized in both the nucleus and cytoplasm (Fig. 3), suggesting a possible shuttling process between these compartments. Similar to the behavior of the Arabidopsis HD2 proteins [39], the two members of soybean HD2 family were localized in the nucleus (Fig. 3). SIR2 proteins were reported to occupy discrete subcellular compartments in plants. In addition, OsSRT1 appears to be mainly localized in the nucleus [66], while SISRT2 localized in both the nucleus and cytoplasm [60] and OsSRT2 and AtSRT2 are found in the mitochondria [67]. In this study, we demonstrated that GmSRT4 localized in the nucleus (Fig. 3). Together, the different subcellular localization patterns of GmHDACs suggest that they might be differentially regulated and may have distinct roles in soybean. However, it is worth noting that the subcellular localization assays described in this study were conducted with Arabidopsis cells so it is entirely possible that soybean-specific interactions could affect the localization pattern in situ.

Increasing evidence suggests that the tissue-specificity and stress-responsiveness of $H D A C$ genes play critical roles in plant development and environmental responses $[14,15,68]$. In our study, quantitative-RT PCR was employed to investigate the tissue-specific and stress-responsive expression patterns of nine typical GmHDACs (Figs. 4 and 5). GmHDA13 was highly expressed in all tissues and developmental stages tested (Fig. 4), which is similar to its close homologous gene AtHDA6. Previous studies have shown that AtHDA6 plays essential roles in various aspects of plant growth and development, such as leaf development and flowering, jasmonate and ethylene signaling, and abiotic stress responses $[17-20,48,49,65,69,70]$. GmHDA13 may thus have functions similar to those of AtHDA6 considering their close evolutionary relationship and their similar expression patterns. GmHDA8, the closest homolog of AtHDA14, is preferentially expressed in cotyledons and leaves (Fig. 4), suggesting a potential role in regulating cotyledon and leaf development. Notably, the expression level of GmSRT2 in leaves was much higher than in other organs, indicating that GmSRT2 might play an important role in regulating leaf development (Fig. 4). In rice, OsSRT1 was reported to regulate leaf senescence and cell death $[66,71]$, however, the functions of SIR2 proteins in plant growth and development remain largely unknown.
Microarray analysis demonstrated that several RPD3 type $H D A C s$ were repressed under high salt and drought treatments in rice [72]. Furthermore, the expression of SlHDACs was induced under salt, dehydration, and low/ high temperature treatments, suggesting that SlHDACs might function in different stress responses in tomato [61]. In this study, we found these nine GmHDACs responded to various abiotic stress treatments, including low/high temperature, flooding, drought, $\mathrm{NaCl}$ and $\mathrm{ABA}$ (Fig. 5). Interestingly, the expression of most genes were repressed under the stress conditions examined, whilst GmSRT2 was significantly induced both by cold and heat treatments (Fig. 5), indicating potentially distinct $H D A C$ gene functions in response to different environmental cues. In Arabidopsis, $\mathrm{ABA}$ and $\mathrm{NaCl}$ treatments are known to repress the gene expression of $A t H D 2 A$, $A t H D 2 B, A t H D 2 C$, and $A t H D 2 D$ [49]. AtHD2C interacts with AtHDA6 and regulates ABA-responsive gene expression by histone deacetylation [49]. Recently, Han et al. (2016) demonstrated that AtHD2D could confer tolerance to abiotic stresses, including drought, salt, and cold stresses in Arabidopsis. In addition, the transcription of HD2 type HDACs is modulated under salt, ABA, and PEG stress treatment in rice [73]. In the present study, GmHDT2 and GmHDT4 were down-regulated following a $\mathrm{NaCl}$ treatment and induced by the application of ABA, which is in support of previous findings (Fig. 5e, f). Furthermore, it has been shown that the expression of $H D A C s$ and the levels of histone $\mathrm{H} 3 \mathrm{~K} 9 \mathrm{ac}$, $\mathrm{H} 4 \mathrm{ac}$ and $\mathrm{H} 4 \mathrm{~K} 5 \mathrm{ac}$ were reduced by low temperature in maize [74]. Similarly, we demonstrated that cold treatment can repress the level of histone $\mathrm{H} 3$ acetylation (H3ac) in soybean while a heat treatment could increased H3ac (Fig. 6), indicating that histone acetylation may play pivotal roles in the plant responses to both cold and heat stress. Interestingly, the status of H3K4me2 and H3K4me3 were also modulated by the cold and heat treatments, indicating histone methyltransferases (HMTs) or histone demethylases (HDMs) may also be involved in these processes (Fig. 6).

\section{Conclusions}

Our work identified $28 H D A C$ genes in soybean, which can be divided into RPD3/HDA1, SIR2, and HD2 families. Segmental duplication events during evolution were the major driver of $H D A C$ gene expansion in soybean. Subcellular localization indicated that GmHDA6, GmHDA13, GmHDA14 and GmHDA16 were localized in both the nucleus and cytoplasm, while GmSRT4, GmHDT2 and GmHDT4 were found solely in the nucleus. Nine typical GmHDAC genes were differentially expressed in all tissues examined and all of them were stress-responsive. Interestingly, our results indicate that global histone acetylation and methylation levels were 
affected in response to cold and heat stress treatments in soybean, indicating that histone modifiers, such as HDACs might be involved in the response of this plant to abiotic stress. Further research is required to determine the function and molecular mechanisms of GmHDACs in plant responses to abiotic stress, which will provide tools for the improvement of soybean productivity.

\section{Methods}

\section{Plant materials and treatments}

Seedlings of soybean (Glycine max) were grown at $25^{\circ} \mathrm{C}$ in a growth cabinet under a light intensity of $10,000 \mathrm{~lx}$ and a $16 \mathrm{~h}$ photoperiod. To investigate tissue or organ specific expression, roots, cotyledons, epicotyls, hypocotyls were harvested from 5-day-old seedlings, while leaves and stems were collected from 2-week-old seedlings. Flowers were collected when they were in full bloom. Seeds were collected 2 weeks after flowering. For the stress treatments, 10-day-old seedlings were exposed to different stress conditions and collected under the light period. Cold and heat stresses were performed by transferring soybean plants grown under control conditions into chambers set at $4{ }^{\circ} \mathrm{C}$ or $42{ }^{\circ} \mathrm{C}$ for the indicated period of time. The flooding treatment was performed by completely submerging seedlings under water at $25^{\circ} \mathrm{C}$. Dehydration was induced by removing plants from the pots and by placing them on filter paper at $25^{\circ} \mathrm{C}$. Soybean seedlings were exposed to salt stress by removing them from soil and by soaking them in a solution containing $250 \mathrm{mM} \mathrm{NaCl}$. For ABA treatment, leaf tissues of the soybean plants were sprayed with $10 \mu \mathrm{M}$ ABA solution. Each treatment was performed on five similar plants (same leaf number, leaf size and plant height) and seedlings without treatment were used as control. After exposure to these stresses for a period of $12 \mathrm{~h}$, the plant tissues were harvested and immediately frozen in liquid nitrogen for further gene expression and protein immunoblotting experiments.

\section{Identification of HDAC genes in soybean}

To identify potential HDACs in soybean, we used the protein sequences of AtHDACs as queries to run the BLASTP program against the soybean database (https://soybase.org/ ). After removing the duplicates from all the captured sequences, we initially retrieved the recognizable domains using BLAST-based NCBI conserved domain searches (https://www.ncbi.nlm.nih.gov/Structure/cdd/ wrpsb.cgi). We then further analyzed these domains using the HMMER-based Simple Modular Architecture Research Tool database (http://smart.embl-heidelberg.de/) and the Pfam software program (http://pfam.xfam.org/search).

\section{Phylogenetic tree construction}

All the HDAC protein sequences from soybean and Arabidopsis were aligned using ClustalW, and the alignment was imported in MEGA5.0 for phylogenetic analysis. The phylogenetic trees were generated using the Maximum Parsimony method with partial deletion. The bootstrap value was assessed with 1000 replicates [75].

\section{Chromosomal localization and gene duplication analyses}

The gene location and chromosome number for each soybean $H D A C$ gene was retrieved from the Phytozome 12 Glycine max Wm82.a2.v1 database (www.phytozome.net). The Circos software was used to determine the chromosomal localization and generate the duplication image of the GmHDAC genes [76]. GmHDAC genes in duplicated genomic regions and the $\mathrm{Ka} / \mathrm{Ks}$ values for the 27 duplicated gene pairs were obtained from Plant Genome Duplication Database (http://chibba.agtec.uga.edu/ duplication/) to evaluate the contribution of tandem and segmental genome duplication to the expansion of the HDAC gene family over evolutionary time. Thus, homologous genes on the same duplicated chromosomal blocks were set as segmental duplication, while two paralogs physically close together were defined as tandem duplications. $\mathrm{Ka} / \mathrm{Ks}$ values superior to 1 indicate positive selection while values below 1 and equal to 1 respectively indicate purifying selection and neural selection [57].

\section{RNA isolation and quantitative real-time RT-PCR}

Total RNAs were isolated from the plant samples using the Hi-Pure Plant RNA Mini Kit (Magen, Guangzhou, China). The first-strand cDNA was generated using the TransScript One-Step gDNA Removal and cDNA synthesis SuperMix (TransGen, Beijing, China). The manufacturers' instructions were followed in each case. Quantitative RT-PCR assays were performed on three biological replicates on a LightCycler 480 system (Roche, Basel, Switzerland) using TransStart Green qPCR SuperMix Kit (TransGen, Beijing, China). The reaction conditions were as follows: $95{ }^{\circ} \mathrm{C}$ for $1 \mathrm{~min}$, followed by 50 cycles of $95{ }^{\circ} \mathrm{C}$ for $10 \mathrm{~s}$, and $60{ }^{\circ} \mathrm{C}$ for $30 \mathrm{~s}$. The soybean CYP2 gene was used as the internal control, and the relative expression levels of genes were calculated using the $2^{-\Delta \Delta C T}$ method [77]. Primer sequences are listed in Additional file 3: Table S2.

\section{Subcellular localization assays}

The subcellular localizations of GmHDACs were first predicted using the pSORT web server (http://www.genscript.com/psort/wolf_psort.html) and confirmed by YFP-tagged transient expression assays in Arabidopsis suspension cultured cells. The full-length coding sequence of GmHDACs was introduced into pSAT6-EYFP-N1 to generate pSAT6-GmHDACs-EYFP, containing a GmHDACs-EYFP 
fusion construct under the control of the CaMV 35S promoter. The fusion constructs and nuclear localization marker NLS-mCherry were co-transfected into protoplast cells for in vivo protein targeting. The protoplast isolation and transient expression were conducted as described previously [78]. After transfection, the protoplasts were incubated at $22{ }^{\circ} \mathrm{C}$ for $12 \mathrm{~h}$ in the dark, and the distribution of the fusion protein was determined using a confocal fluorescence microscope.

\section{Protein immunoblotting}

Soybean leaves sampled from control, cold $\left(4^{\circ} \mathrm{C}\right)$ and heat $\left(42{ }^{\circ} \mathrm{C}\right)$ treated seedlings were ground to a powder in liquid nitrogen and mixed with $1 \mathrm{~mL}$ of ice-cold extraction buffer (0.1 M Tris-HCl, pH 8.0, 10\% glycol, 3\% SDS, $0.05 \%$ beta-mercaptoethanol). The samples were then boiled for $5 \mathrm{~min}$, centrifuged at 13,000 rpm for $10 \mathrm{~min}$ and the supernatants collected as the total protein fractions. The protein were mixed with a loading dye and loaded on $12 \%$ polyacrylamide gels. After electrophoresis and transfer to a PVDF membrane, the samples were immunoblotted with the following commercial antibodies: anti-H3 (Millipore, 05-499), anti-H3ac (Millipore, 06-599), anti-H3K4me2 (Millipore, 07-030) and anti-H3K4me3 (Millipore, 04-745). The experiments were carried out three times and the Image J software was used to quantify the relative protein levels.

\section{Statistical analyses}

A Student's $t$-test (two tail, unpaired, equal variance) was used to determine the statistical significance of the differential transcripts abundance patterns and protein accumulation levels between treatments and their corresponding controls. Differential expression data were regarded as statistically significant and were marked by * only when passing the $t$-test with a $P$-value of $<0.05$.

\section{Additional files}

Additional file 1: Table S1. Segmental duplication events of soybean genes during evolution. (DOC $67 \mathrm{~kb}$ )

Additional file 2: Figure S1. Expression profiles of GMHDAC genes in developmental seeds. The transcript profiling data of soybean seeds was extracted from the publicly-available Soybase database (https:/www.soybase.org/ for heatmap generation. The colors indicate expression intensity (red, high expression; black, low expression; grey, no expression). (JPG 538 kb)

Additional file 3: Table S2. Primers used in this study. (DOC $50 \mathrm{~kb}$ )

\section{Abbreviations}

ABA: Abscisic acid; bp: base pair; CaMv: Cauliflower mosaic virus; cDNA: complementary deoxyribonucleicacid; DAF: days after flowering; gDNA: genomic deoxyribonucleicacid; kDa: kilo Dalton; NLS: nuclear localization signal; RNA: ribonucleicacid; RT-PCR: Real time-polymerase chain reaction; YFP: Yellow fluorescent protein

\section{Acknowledgements}

We thank editors and reviewers for the careful reading and valuable comments. We apologize to researchers whose studies are not cited due to space limitations.

\section{Funding}

This work was financially supported by Youth Innovation Promotion Association, Chinese Academy of Sciences (2017399), Guangdong Natural Science Foundation (2018A030313350), the Strategic Priority Research Program of Chinese Academy of Sciences (XDA13020603), Innovation Training Programs for Undergraduates (CAS), the National Natural Science Foundation of China (31671467 and 31770048) and the China 1000-Talents Plan for young researchers (C83025).

\section{Availability of data and materials}

The datasets generated and analysed during the current study are available from the corresponding author on reasonable request.

\section{Authors' contributions}

$M L, C G$ and $J L$ conceived and designed the experiments; $C Y, W S, H C, L C, Y X$, $X Z, C L, C C, J Z, Q L$ and $M L$ performed the experiments; ML, CY and CG analysed the data; $M L$ and $C Y$ wrote the manuscript; $C Y, L C, Y X, X Z$, JC and $M L$ revised the manuscript; all authors read and approved the final manuscript.

\section{Ethics approval and consent to participate}

The plant materials were collected from South China Botanical Garden, CAS. The experimental research on plants, including collection of plant material, was complied with the institutional, national, or international guidelines.

\section{Consent for publication}

Not applicable.

\section{Competing interests}

The authors declare that they have no competing interests.

\section{Publisher's Note}

Springer Nature remains neutral with regard to jurisdictional claims in published maps and institutional affiliations.

\section{Author details}

${ }^{1}$ Guangdong Provincial Key Laboratory of Applied Botany, Key Laboratory of South China Agricultural Plant Molecular Analysis and Genetic Improvement, South China Botanical Garden, Chinese Academy of Sciences, Guangzhou 510650, China. ${ }^{2}$ Guangdong Provincial Key Laboratory of Biotechnology for Plant Development, School of Life Sciences, South China Normal University, Guangzhou 510631, China. ${ }^{3}$ University of Chinese Academy of Sciences, Beijing 100049, China. ${ }^{4}$ Institute for Food and Bioresource Engineering, Department of Energy and Resources Engineering and BIC-ESAT, College of Engineering, Peking University, Beijing 100871, China. ${ }^{5}$ Key Laboratory of Crop Genetics and Physiology of Jiangsu Province/Key Laboratory of Plant Functional Genomics of the Ministry of Education, College of Agriculture, Yangzhou University, Yangzhou 225009, China. ${ }^{6}$ Department of Plant Science, McGill University, Sainte-Anne-de-Bellevue, QC, Canada.

Received: 23 November 2017 Accepted: 1 October 2018

Published online: 11 October 2018

\section{References}

1. Wang W, Vinocur B, Altman A. Plant responses to drought, salinity and extreme temperatures: towards genetic engineering for stress tolerance. Planta. 2003;218(1):1-14.

2. Qin F, Shinozaki K, Yamaguchi-Shinozaki K. Achievements and challenges in understanding plant abiotic stress responses and tolerance. Plant Cell Physiol. 2011;52(9):1569-82.

3. Deshmukh R, Sonah H, Patil G, Chen W, Prince S, Mutava R, Vuong T, Valliyodan B, Nguyen HT. Integrating omic approaches for abiotic stress tolerance in soybean. Front Plant Sci. 2014;5:244.

4. Luo M, Liu X, Singh P, Cui Y, Zimmerli L, Wu K. Chromatin modifications and remodeling in plant abiotic stress responses. Biochimica et Biophysica Acta (BBA) - Gene Regulatory Mechanisms. 2012;1819(2):129-36. 
5. Zong W, Zhong X, You J, Xiong L. Genome-wide profiling of histone H3K4tri-methylation and gene expression in rice under drought stress. Plant Mol Biol. 2013;81(1):175-88.

6. Kim JM, Sasaki T, Ueda M, Sako K, Seki M. Chromatin changes in response to drought, salinity, heat, and cold stresses in plants. Front Plant Sci. 2015;6:114.

7. Mehdi S, Derkacheva M, Ramstr M, Kralemann L, Bergquist J, Hennig L. The WD40 domain protein MSI1 functions in a histone deacetylase complex to fine-tune abscisic acid signaling. Plant Cell. 2016;28(1):42-54.

8. Lusser A, Brosch G, Loidl A, Haas H, Loidl P. Identification of maize histone deacetylase HD2 as an acidic nucleolar phosphoprotein. Science. 1997; 277(5322):88-91.

9. Yang XJ, Seto E. HATs and HDACs: from structure, function and regulation to novel strategies for therapy and prevention. Oncogene. 2007;26:5310.

10. Pandey R, Mler A, Napoli CA, Selinger DA, Pikaard CS, Richards EJ, Bender J, Mount DW, Jorgensen RA. Analysis of histone acetyltransferase and histone deacetylase families of Arabidopsis thaliana suggests functional diversification of chromatin modification among multicellular eukaryotes. Nucleic Acids Res. 2002;30(23):5036-55.

11. Haigis MC, Guarente LP. Mammalian sirtuins--emerging roles in physiology, aging, and calorie restriction. Genes Dev. 2006;20(21):2913-21.

12. Lee $\mathrm{WK}$, Cho $\mathrm{MH}$. Telomere-binding protein regulates the chromosome ends through the interaction with histone deacetylases in Arabidopsis thaliana. Nucleic Acids Res. 2016:44(10):4610-24.

13. Alinsug MV, Yu CW, Wu K. Phylogenetic analysis, subcellular localization, and expression patterns of RPD3/HDA1 family histone deacetylases in plants. BMC Plant Biol. 2009;9(1):37.

14. Ma X, LV S, Zhang C, Yang C. Histone deacetylases and their functions in plants. Plant Cell Rep. 2013;32(4):465-78.

15. Liu X, Yang S, Zhao M, Luo M, Yu CW, Chen CY, Tai R, Wu K. Transcriptional repression by histone deacetylases in plants. Mol Plant. 2014;7(5):764-72.

16. Aufsatz W, Mette MF, van der Winden J, Matzke M, Matzke AJM: HDA6, a putative histone deacetylase needed to enhance DNA methylation induced by double-stranded RNA. EMBO J 2002, 21(24):6832-6841.

17. Probst AV, Fagard M, Proux F, Mourrain P, Boutet S, Earley K, Lawrence RJ, Pikaard CS, Murfett J, Furner l, et al. Arabidopsis histone deacetylase HDA6 is required for maintenance of transcriptional gene silencing and determines nuclear organization of rDNA repeats. Plant Cell. 2004;16(4): 1021-34.

18. Yu CW, Liu X, Luo M, Chen C, Lin X, Tian G, Lu Q, Cui Y, Wu K. HISTONE DEACETYLASE6 interacts with FLOWERING LOCUS D and regulates flowering in Arabidopsis. Plant Physiol. 2011;156(1):173-84.

19. Zhu Z, An F, Feng Y, Li P, Xue L, A M JZ, Kim JM, To TK, Li W, et al, Derepression of ethylene-stabilized transcription factors (EIN3/EIL1) mediates jasmonate and ethylene signaling synergy in Arabidopsis. Proc Natl Acad Sci. 2011;108(30):12539-44.

20. Luo M, Yu CW, Chen FF, Zhao L, Tian G, Liu X, Cui Y, Yang JY, Wu K. Histone deacetylase HDA6 is functionally associated with AS1 in repression of KNOX genes in Arabidopsis. PLoS Genet. 2012;8(12):e1003114.

21. Wang L, Kim J, Somers DE. Transcriptional corepressor TOPLESS complexes with pseudoresponse regulator proteins and histone deacetylases to regulate circadian transcription. Proc Natl Acad Sci. 2013;110(2):761-6.

22. Tian L, Chen ZJ. Blocking histone deacetylation in Arabidopsis induces pleiotropic effects on plant gene regulation and development. Proc Natl Acad Sci. 2001;98(1):200-5.

23. Xu CR, Liu C, Wang YL, Li LC, Chen WQ, Xu ZH, Bai SN. Histone acetylation affects expression of cellular patterning genes in the Arabidopsis root epidermis. Proc Natl Acad Sci. 2005;102(40):14469-74.

24. Long JA, Ohno C, Smith ZR, Meyerowitz EM. TOPLESS regulates apical embryonic fate in Arabidopsis. Science. 2006;312(5779):1520-3.

25. Krogan NT, Hogan K, Long JA. APETALA2 negatively regulates multiple floral organ identity genes in Arabidopsis by recruiting the co-repressor TOPLESS and the histone deacetylase HDA19. Development. 2012;139(22):4180-90.

26. Tran HT, Nimick M, Uhrig RG, Templeton G, Morrice N, Gourlay R, DeLong A, Moorhead GBG. Arabidopsis thaliana histone deacetylase 14 (HDA14) is an a-tubulin deacetylase that associates with PP2A and enriches in the microtubule fraction with the putative histone acetyltransferase ELP3. Plant J. $2012 ; 71(2): 263-72$

27. Cigliano RA, Cremona G, Paparo R, Termolino P, Perrella G, Gutzat R, Consiglio MF, Conicella C. Histone deacetylase AtHDA7 is required for female gametophyte and embryo development in Arabidopsis. Plant Physiol. 2013;163(1):431-40
28. Kim W, Latrasse D, Servet C, Zhou DX. Arabidopsis histone deacetylase HDA9 regulates flowering time through repression of AGL19. Biochem Biophys Res Commun. 2013;432(2):394-8.

29. Liu C, Li LC, Chen WQ, Chen X, Xu ZH, Bai SN. HDA18 affects cell fate in Arabidopsis root epidermis via histone acetylation at four kinase genes. Plant Cell. 2013;25(1):257-69.

30. Liu X, Chen CY, Wang K-C, Luo M, Tai R, Yuan L, Zhao M, Yang S, Tian G, Cui $Y$, et al. PHYTOCHROME INTERACTING FACTOR3 associates with the histone deacetylase HDA15 in repression of chlorophyll biosynthesis and photosynthesis in etiolated Arabidopsis seedlings. Plant Cell. 2013; 25(4):1258-73.

31. Zhou Y, Tan B, Luo M, Li Y, Liu C, Chen C, Yu CW, Yang S, Dong S, Ruan J, et al. HISTONE DEACETYLASE19 interacts with HSL1 and participates in the repression of seed maturation genes in Arabidopsis seedlings. Plant Cell. 2013;25(1):134-48.

32. Ryu $\mathrm{H}$, Cho H, Bae W, Hwang I. Control of early seedling development by BES1/TPL/HDA19-mediated epigenetic regulation of ABI3. Nat Commun. 2014;5:4138

33. Chen CY, Wu K, Schmidt W. The histone deacetylase HDA19 controls root cell elongation and modulates a subset of phosphate starvation responses in Arabidopsis. Sci Rep. 2015:5:15708.

34. Luo M, Tai R, Yu CW, Yang S, Chen CY, Lin WD, Schmidt W, Wu K. Regulation of flowering time by the histone deacetylase HDA5 in Arabidopsis. Plant J. 2015;82(6):925-36.

35. Gu D, Chen CY, Zhao M, Zhao L, Duan X, Duan J, Wu K, Liu X. Identification of HDA15-PIF1 as a key repression module directing the transcriptional network of seed germination in the dark. Nucleic Acids Res. 2017:45(12):7137-50.

36. Tang $Y$, Liu X, Liu X, Li Y, Wu K, Hou X. Arabidopsis NF-YCs mediate the light-controlled hypocotyl elongation via modulating histone acetylation. Mol Plant. 2017;10(2):260-73.

37. Gao MJ, Li X, Huang J, Gropp GM, Gjetvaj B, Lindsay DL, Wei S, Coutu C, Chen Z, Wan XC, et al. SCARECROW-LIKE15 interacts with HISTONE DEACETYLASE19 and is essential for repressing the seed maturation programme. Nat Commun. 2015;6:7243.

38. Wu K, Tian L, Malik K, Brown D, Miki B. Functional analysis of HD2 histone deacetylase homologues in Arabidopsis thaliana. Plant J. 2000;22(1):19-27.

39. Zhou C, Labbe H, Sridha S, Wang L, Tian L, Latoszek-Green M, Yang Z, Brown D, Miki B, Wu K. Expression and function of HD2-type histone deacetylases in Arabidopsis development. Plant J. 2004;38(5):715-24.

40. Farhi J, Tian G, Fang H, Maxwell D, Xing T, Tian L. Histone deacetylase HD2D is involved in regulating plant development and flowering time in Arabidopsis. Plant Signal Behav. 2017;12(7):e1300742.

41. Li H, Torres-Garcia J, latrasse D, Benhamed M, Schilderink S, Zhou W, Kulikova O, Hirt H, Bisseling T: Plant-specific histone deacetylases HDT?Regulate GIBBERELLIN 2-OXIDASE 2 expression to control Arabidopsis root meristem cell number. Plant Cell 2017, 29:tpc. 2017:00366.

42. Ueno Y, Ishikawa T, Watanabe K, Terakura S, Iwakawa H, Okada K, Machida C, Machida Y. Histone deacetylases and ASYMMETRIC LEAVES2 are involved in the establishment of polarity in LEAVES of Arabidopsis. Plant Cell. 2007; 19(2):445-57.

43. Wang C, Gao F, Wu J, Dai J, Wei C, Li Y. Arabidopsis putative deacetylase AtSRT2 regulates basal defense by suppressing PAD4, EDS5 and SID2 expression. Plant Cell Physiol. 2010;51(8):1291-9.

44. Zhang H, Lu Y, Zhao Y, Zhou DX. OsSRT1 is involved in rice seed development through regulation of starch metabolism gene expression. Plant Sci. 2016;248:28-36.

45. Zhou C, Zhang L, Duan J, Miki B, Wu K. HISTONE DEACETYLASE19 is involved in Jasmonic acid and ethylene signaling of pathogen response in Arabidopsis. Plant Cell. 2005;17(4):1196-204

46. Kim KC, Lai Z, Fan B, Chen Z. Arabidopsis WRKY38 and WRKY62 transcription factors interact with histone deacetylase 19 in basal defense. Plant Cell. 2008;20(9):2357-71.

47. Chen LT, Wu K. Role of histone deacetylases HDA6 and HDA19 in ABA and abiotic stress response. Plant Signal Behav. 2010;5(10):1318-20.

48. To TK, Nakaminami K, Kim JM, Morosawa T, Ishida J, Tanaka M, Yokoyama S, Shinozaki K, Seki M. Arabidopsis HDA6 is required for freezing tolerance. Biochem Biophys Res Commun. 2011:406(3):414-9.

49. Luo M, Wang YY, Liu X, Yang S, Lu Q, Cui Y, Wu K. HD2C interacts with HDA6 and is involved in ABA and salt stress response in Arabidopsis. J Exp Bot. 2012;63(8):3297-306 
50. Jung JH, Park JH, Lee S, To TK, Kim JM, Seki M, Park CM. The cold signaling attenuator HIGH EXPRESSION OF OSMOTICALLY RESPONSIVE GENE1 activates FLOWERING LOCUS C transcription via chromatin remodeling under short-term cold stress in Arabidopsis. Plant Cell. 2013;25(11):4378-90.

51. Buszewicz D, Archacki R, Palusiński A, Kotliński M, Fogtman A, IwanickaNowicka R, Sosnowska K, Kuciński J, Pupel P, Olędzki J, et al. HD2C histone deacetylase and a SWI/SNF chromatin remodelling complex interact and both are involved in mediating the heat stress response in Arabidopsis. Plant Cell Environ. 2016;39(10):2108-22.

52. Zheng $Y$, Ding $Y$, Sun $X$, Xie S, Wang D, Liu X, Su L, Wei W, Pan L, Zhou DX. Histone deacetylase HDA9 negatively regulates salt and drought stress responsiveness in Arabidopsis. J Exp Bot. 2016;67(6):1703-13.

53. Ueda M, Matsui A, Tanaka M, Nakamura T, Abe T, Sako K, Sasaki T, Kim JM, Ito A, Nishino N, et al. The distinct roles of class I and II RPD3-like histone deacetylases in salinity stress response. Plant Physiol. 2017;175:1760-73.

54. Sridha S, Wu K. Identification of AtHD2C as a novel regulator of abscisic acid responses in Arabidopsis. Plant J. 2006;46(1):124-33.

55. Han Z, Yu H, Zhao Z, Hunter D, Luo X, Duan J, Tian L. AtHD2D gene plays a role in plant growth, development, and response to abiotic stresses in Arabidopsis thaliana. Front Plant Sci. 2016;7:310.

56. Cannon SB, Mitra A, Baumgarten A, Young ND, May G. The roles of segmental and tandem gene duplication in the evolution of large gene families in Arabidopsis thaliana. BMC Plant Biol. 2004:4:10.

57. Yang Z, Nielsen R. Estimating synonymous and nonsynonymous substitution rates under realistic evolutionary models. Mol Biol Evol. 2000;17:32-43.

58. Fu W, Wu K, Duan J. Sequence and expression analysis of histone deacetylases in rice. Biochem Biophys Res Commun. 2007;356(4):843-50.

59. Aquea F, Timmermann T, Arce-Johnson P. Analysis of histone acetyltransferase and deacetylase families of Vitis vinifera. Plant Physiol Biochem. 2010;48(23):194-9.

60. Zhao L, Lu J, Zhang J, Wu PY, Yang S, Wu K. Identification and characterization of histone deacetylases in tomato (Solanum lycopersicum). Front Plant Sci. 2014;5:760

61. Guo JE, Hu Z, Guo X, Zhang L, Yu X, Zhou S, Chen G. Molecular characterization of nine tissue-specific or stress-responsive genes of histone deacetylase in tomato (Solanum lycopersicum). J Plant Growth Regul. 2017; 36(3):566-77.

62. Gray SG, Ekstr TJ. The human histone deacetylase family. Exp Cell Res. 2001; 262(2):75-83.

63. Alinsug MV, Chen FF, Luo M, Tai R, Jiang L, Wu K. Subcellular localization of class II HDAs in Arabidopsis thaliana: nucleocytoplasmic shuttling of HDA15 is driven by light. PLoS One. 2012;7(2):e30846.

64. Earley K, Lawrence RJ, Pontes O, Reuther R, Enciso AJ, Silva M, Neves N, Gross M, Viegas W, Pikaard CS. Erasure of histone acetylation by Arabidopsis HDA6 mediates large-scale gene silencing in nucleolar dominance. Genes Dev. 2006;20(10):1283-93.

65. Wu K, Zhang L, Zhou C, Yu CW, Chaikam V. HDA6 is required for jasmonate response, senescence and flowering in Arabidopsis. J Exp Bot. 2008;59(2): 225-34.

66. Huang L, Sun Q, Qin F, Li C, Zhao Y, Zhou DX. Down-regulation of a SILENT INFORMATION REGULATOR2-related histone deacetylase gene, OsSRT1, induces DNA fragmentation and cell death in Rice. Plant Physiol. 2007; 144(3):1508-19.

67. König AC, Hartl M, Pham PA, Laxa M, Boersema PJ, Orwat A, Kalitventseva I, PI hinger M, Braun HP, Leister $D$, et al. The Arabidopsis class II Sirtuin is a lysine deacetylase and interacts with mitochondrial energy metabolism. Plant Physiol. 2014;164(3):1401-14.

68. Yuan L, Liu X, Luo M, Yang S, Wu K. Involvement of histone modifications in plant abiotic stress responses. J Integr Plant Biol. 2013;55(10):892-901.

69. Tanaka M, Kikuchi A, Kamada H. The Arabidopsis histone deacetylases HDA6 and HDA19 contribute to the repression of embryonic properties after germination. Plant Physiol. 2008;146(1):149-61.

70. Chen LT, Luo M, Wang YY, Wu K. Involvement of Arabidopsis histone deacetylase HDA6 in ABA and salt stress response. J Exp Bot. 2010;61(12): 3345-53.

71. Fang C, Zhang H, Wan J, Wu Y, Li K, Jin C, Chen W, Wang S, Wang W, Zhang H, et al. Control of leaf senescence by an $\mathrm{MeOH}$-Jasmonates Cascade that is epigenetically regulated by OsSRT1 in Rice. Mol Plant. 2016;9(10):1366-78.

72. Hu Y, Qin F, Huang L, Sun Q, Li C, Zhao Y, Zhou DX. Rice histone deacetylase genes display specific expression patterns and developmental functions. Biochem Biophys Res Commun. 2009;388(2):266-71.
73. Zhao J, Zhang J, Zhang W, Wu K, Zheng F, Tian L, Liu X, Duan J. Expression and functional analysis of the plant-specific histone deacetylase HDT701 in rice. Front Plant Sci. 2014;5:764.

74. Hu Y, Zhang L, Zhao L, Li J, He S, Zhou K, Yang F, Huang M, Jiang L, Li L. Trichostatin a selectively suppresses the cold-induced transcription of the ZmDREB1 gene in maize. PLoS One. 2011;6(7):e22132.

75. Tamura K, Peterson D, Peterson N, Stecher G, Nei M, Kumar S. MEGA5: molecular evolutionary genetics analysis using maximum likelihood, evolutionary distance, and maximum parsimony methods. Mol Biol Evol. 2011;28(10):2731-9.

76. Krzywinski M, Schein J, Birol I, Connors J, Gascoyne R, Horsman D, Jones SJ, Marra MA. Circos: an information aesthetic for comparative genomics. Genome Res. 2009;19(9):1639-45.

77. Livak KJ, Schmittgen TD. Analysis of relative gene expression data using real-time quantitative PCR and the $2^{-\Delta \Delta C T}$ method. Methods. 2001;25(4): 402-8.

78. Miao Y, Jiang L. Transient expression of fluorescent fusion proteins in protoplasts of suspension cultured cells. Nat Protoc. 2007;2:2348.
Ready to submit your research? Choose BMC and benefit from:

- fast, convenient online submission

- thorough peer review by experienced researchers in your field

- rapid publication on acceptance

- support for research data, including large and complex data types

- gold Open Access which fosters wider collaboration and increased citations

- maximum visibility for your research: over $100 \mathrm{M}$ website views per year

At BMC, research is always in progress.

Learn more biomedcentral.com/submissions 